\title{
Depth Analysis of Crystalline Silicon Used for Radiation-Hard Detectors
}

\author{
S.J. Moloi ${ }^{a, *}$ AND M. MCPherson ${ }^{b}$ \\ ${ }^{a}$ Department of Physics, University of South Africa, P.O. Box 392, Pretoria 0003, South Africa \\ ${ }^{b}$ Centre for Postgraduate Studies, Cape Peninsula University of Technology, P.O. Box 652, \\ Cape Town 8000, South Africa \\ (Received 17 March, 2017; in final form 8 August, 2017)
}

\begin{abstract}
Depth analysis of metal-doped crystalline silicon by the X-ray photoelectron spectroscopy technique is presented in this work. The results from this technique are used to complement those from previous techniques. The metals diffused into the silicon are gold, platinum, erbium, and niobium. In silicon, these metals induce defects that are responsible for relaxation behaviour of the material. Relaxation material is radiation-hard since the effects of radiation on devices fabricated on the material are suppressed. Considerable amounts of gold, platinum, and niobium are found in the silicon bulk. The results of this work are in good agreement with those reported earlier on the same samples using the Rutherford backscattering technique. The spectra of the natural contaminants, carbon and oxygen, are also analysed in this work.
\end{abstract}

DOI: 10.12693/APhysPolA.132.1387

PACS/topics: silicon, diffusion, XPS, detectors

\section{Introduction}

Crystalline silicon needs to be modified in the bulk in order to improve the properties of devices fabricated on the material. The properties have to be improved since devices fabricated on the material fail to operate efficiently as radiation detectors. The failure to operate efficiently is due to damage that occurs in the material by incident high energetic particles $[1,2]$. As they become incident onto the material, the particles penetrate the material and collide with the semiconductor lattice. The incident particles transfer sufficient energy to the silicon to displace an atom within the lattice out of its normal position such that interstitial atoms and vacancies are generated in the bulk of the material [3]. The result is that the lattice structure of the material changes and this degrades the performance of the devices.

In order to improve the properties of the devices, the silicon material can be doped with metals such as gold, platinum, erbium, or niobium [4-11]. These metals are responsible for a change in material behaviour from lifetime to relaxation [9-11]. In relaxation material, $\tau_{D} \gg \tau_{0}$ while in lifetime material, $\tau_{D} \ll \tau_{0}[12-15]$. Here, $\tau_{D}$ is the dielectric relaxation time and $\tau_{0}$ is the minority carrier recombination lifetime [12-15]. In relaxation material the effects of radiation are suppressed $[5,9]$ such that the damage is minimal. In this case the Fermi level of the material is not affected by any incident radiation [16]. This is because the Fermi level in relaxation material becomes pinned at midgap [17].

Depending on their nature and their amount in silicon, the metals can generate defects that have characteristics

*corresponding author; e-mail: moloisj@unisa.ac.za similar to those generated by radiation [11]. Thus, the metals can render the material useless to fabricate devices that can be used as radiation detectors. It is, therefore, very important that the concentration of metal required to cause relaxation behaviour in the material is known. It is also essential that diffusion mechanisms of these metals into silicon are investigated so that their undesired effects are minimized while the desired effects are improved.

In studying the diffusion mechanisms of gold and platinum into silicon, it has been found that the diffused atoms occupy both substitutional and interstitial sites $[4,6]$. The interchange of atoms between the sites during diffusion is controlled by the dissociative and the kick-out mechanisms $[4,6]$. In the former process the diffusion involves silicon vacancies, while in the latter one the diffusion involves self-interstitials. The diffusion mechanisms of erbium and niobium into silicon need to be studied as well, since they exhibit similar effects as those of gold and platinum.

In this work, depth analysis by X-ray photoelectron spectroscopy (XPS) was carried out on metal-coated $p$ type silicon prior to, and after, metal diffusion. The spectrum peaks generated from the metal-coated samples are found to be reduced and shifted towards higher sputter depth regions which indicates that the metals are found in the silicon bulk after the diffusion process. The spectra peaks of the gold, platinum, and niobium-coated samples are found to be higher than those of silicon after the diffusion process. This indicates that the concentration of the metals is higher than that of silicon at the depth of $20 \mathrm{~nm}$ and $10 \mathrm{~nm}$ in case of niobium. These results are in agreement with those obtained earlier from the Rutherford backscattering spectroscopy (RBS) technique [18]. However, in the work reported before based on RBS results, the diffusion was evaluated by noting a change in spectra after the annealing process. These metals, dif- 
fused in the bulk, convert silicon from a lifetime to a relaxation material [11].

\section{Experimental technique}

XPS measurements were carried out on the metalcoated $p$-type silicon samples prior to, and after, the diffusion process. The measurements were done using the Physical Electronics Quantum (PEQ) 2000 instrument at the Council for Scientific and Industrial Research (CSIR) in South Africa. The samples were annealed at $900^{\circ} \mathrm{C}$ for $18 \mathrm{~h}$ to allow diffusion of the metals into the silicon bulk. $\mathrm{An} \mathrm{Al} K_{\alpha}$ X-ray source with energy of $1486.6 \mathrm{eV}$ was used to excite electrons from the surface of the sample to an entrance slit of hemisphere analyser. The diameter of the $\mathrm{X}$-ray beam was $100 \mu \mathrm{m}$. The mean analysing energy of the analyser was $58.7 \mathrm{eV}$. This meant that only electrons of this kinetic energy will be detected. The detection angle was set at $45^{\circ}$ from a surface that is normal to the sample. For depth profiling measurements, the sample surfaces were sputtered with $2.0 \mathrm{keV}$ argon ions while the binding energy regions of the specific element were being monitored. The measurements were performed from the top surface to the bulk of the sample and were carried out at room temperature and at vacuum pressures below $10^{-9}$ mbar. A detailed description of the samples and their preparations has been outlined elsewhere [19].

\section{Results and discussion}

The XPS spectra of silicon, gold, platinum, erbium and niobium are presented here. Carbon and oxygen are the main contaminants of the samples and their spectra are also presented but not thoroughly discussed as they are not for our concern. The spectra are presented in the following sections with the relative atomic concentration as a function of the sputter depth.

\subsection{Gold-deposited samples}

Figure 1 shows the XPS spectra for gold-deposited samples (a) prior to and (b) after the diffusion process. In Fig. 1a the spectra show that carbon with $22 \%$ atomic concentration and oxygen with $75 \%$ atomic concentration dominate on the surface of the silicon (depth of $0 \mathrm{~nm}$ ). A sudden decrease in concentration is observed for both elements and they disappear completely at a depth of $4 \mathrm{~nm}$. Since the two elements are found only at the surface, they do not have an effect in the bulk and this is why they are not for our concern for this sample.

Gold is not observed on the surface (at a depth of $0 \mathrm{~nm}$ ). This means that a layer of carbon and oxygen covered the gold surface after deposition. Gold with atomic concentration of $100 \%$ is observed at a depth of $5 \mathrm{~nm}$. The concentration of gold decreases until it becomes zero at $18 \mathrm{~nm}$. The atomic concentration of silicon increases sharply to $98 \%$ and becomes almost constant up to a depth of $30 \mathrm{~nm}$. An increase in silicon concentration corresponds to a decrease in gold concentration. This shows that there is no interface layer formed between gold and silicon [19]. It is evident from these results that at depths greater than $18 \mathrm{~nm}$ only silicon atoms dominate.
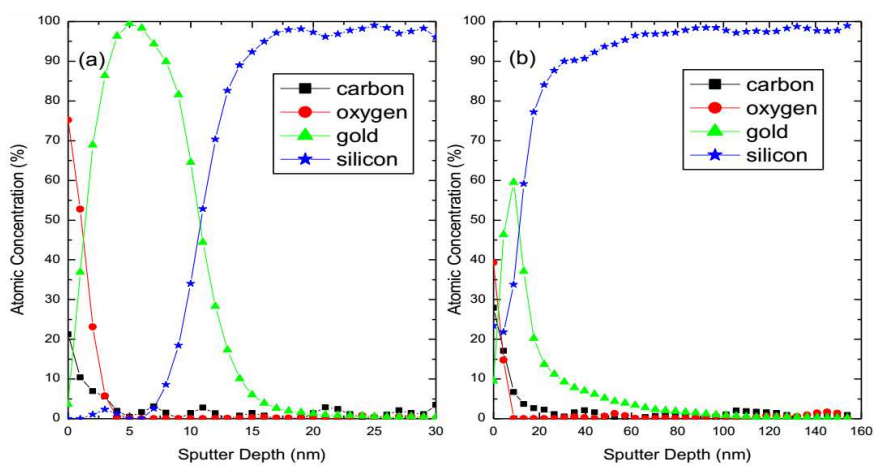

Fig. 1. XPS depth profile for gold-deposited samples prior to (a) and after (b) the diffusion process.

In Fig. 1b the spectra show carbon with $40 \%$ atomic concentration and oxygen with $28 \%$ atomic concentration on the surface. The amount of these contaminants decreases significantly with depth and disappears at $10 \mathrm{~nm}$ and $23 \mathrm{~nm}$, respectively. These results show that carbon and oxygen do not diffuse into the bulk. Gold is also found on the surface with a $10 \%$ atomic concentration and this shows that not all the gold deposited is diffused into the silicon. Gold is seen deep into the bulk at depths of over $80 \mathrm{~nm}$.

Silicon is also observed with $26 \%$ atomic concentration on the surface of the samples after the diffusion process. The concentration decreases slightly before a depth of $7 \mathrm{~nm}$ and then increases sharply to $90 \%$ at $25 \mathrm{~nm}$. After a depth of $26 \mathrm{~nm}$ the spectrum shows a tendency to almost saturate. This sharp decrease of gold concentration that corresponds to a sharp increase of silicon concentration indicates that there is no interface layer formed between gold and silicon after the diffusion process. The presence of silicon on the surface of the sample shows that silicon was exposed when gold was diffusing into the bulk. There is indication of a good mix of gold and silicon up to depths of $80 \mathrm{~nm}$.

The gold peak has been reduced from $100 \%$ to $60 \%$ and shifted from $5 \mathrm{~nm}$ to $18 \mathrm{~nm}$ after diffusion. The reduction of the peak indicates that the concentration of gold has been reduced on the surface while the shift of the peak to a higher sputter depth indicates that gold has diffused into the bulk of the silicon.

\subsection{Platinum-deposited samples}

Figure 2 shows the XPS spectra for the platinumdeposited samples (a) prior to and (b) after the diffusion process. The spectra presented in Fig. 2a show that carbon with $55 \%$ atomic concentration and oxygen with $14 \%$ atomic concentration dominates on the surface of the silicon. A decrease in the concentration of carbon to $47 \%$ is observed after a depth of $1 \mathrm{~nm}$ and then it almost remains constant up to a depth of $8.5 \mathrm{~nm}$. At a depth of $9 \mathrm{~nm}$ the concentration decreases and becomes $0 \%$ after the sputter depth of $15 \mathrm{~nm}$. Since the concentration is not decreasing sharply at a depth lower than $10 \mathrm{~nm}$ 
as observed in Fig. 1a for gold-deposited samples, these results show that there is a thick layer of carbon on the surface of the silicon.

The oxygen spectrum, on the other hand, disappears completely after a depth of $2 \mathrm{~nm}$ to show that oxygen is very thin on the surface of the silicon. The $22 \%$ atomic concentration of platinum is also found on the surface of the silicon indicating that platinum is not completely covered by carbon and oxygen layers. Platinum concentration increases sharply to $55 \%$ at a depth of $1.5 \mathrm{~nm}$ and then becomes almost constant up to a depth of $8.5 \mathrm{~nm}$. After a depth of $9 \mathrm{~nm}$ the platinum spectrum follows the one of carbon and showing a sharp decrease in concentration to $4 \%$ at $16 \mathrm{~nm}$. Carbon and platinum might be bonded to each other since their spectra reach the saturation and show a sharp decrease in concentration at the same depth.

These results explain the small peak that was observed in the RBS results for the platinum-deposited sample [18]. This sample was contaminated by carbon during sample preparation since the peak was also observed [18] in the RBS result that were obtained immediately after the deposition. The spectrum in Fig. 2a shows silicon with $9 \%$ atomic concentration on the surface which decreases rapidly until it becomes $0 \%$ at a depth of $6 \mathrm{~nm}$. After $7.5 \mathrm{~nm}$ the silicon concentration increases sharply to $92 \%$ at $17.5 \mathrm{~nm}$ and then becomes constant up to $30 \mathrm{~nm}$. The sharp increase of silicon concentration corresponds to the sharp decrease of carbon and platinum concentrations to show that there is no interface layer formed between platinum and silicon. Even though they are bonded, carbon and platinum are not found in the silicon bulk. This is shown in Fig. 2a where silicon atoms dominate after $18 \mathrm{~nm}$.
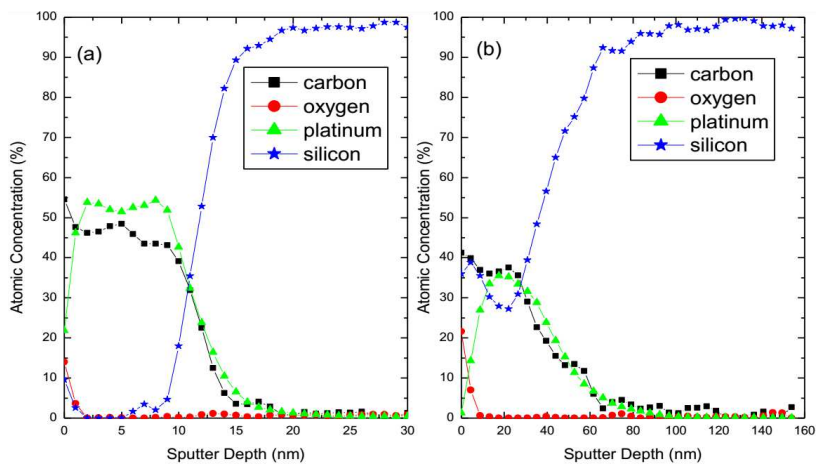

Fig. 2. XPS depth profile for platinum-deposited samples prior to (a) and after the (b) diffusion process.

In Fig. 2b spectra show carbon with $41 \%$ atomic concentration on the surface and it remains almost constant up to a depth of $30 \mathrm{~nm}$. After a depth of $31 \mathrm{~nm}$, the carbon concentration decreases with depth. Comparing Fig. $2 \mathrm{a}$ and $2 \mathrm{~b}$, it can be noted that in Fig. $2 \mathrm{~b}$ the carbon concentration has decreased at low depth. This is an indication that carbon concentration has decreased on the surface and diffused into the silicon. As it was observed in
RBS results [18], the shifting of small peak towards the lower energy indicates that carbon atoms diffused into the silicon. The oxygen spectrum, on the other hand, shows $22 \%$ atomic concentration on the surface. Since the layer is very thin on the surface, the concentration decreases and becomes $0 \%$ at low depth.

Platinum is found on the surface with $2 \%$ atomic concentration. The concentration increases to $35 \%$ at $20 \mathrm{~nm}$. After $21 \mathrm{~nm}$ the concentration decreases rapidly to $0 \%$ at $70 \mathrm{~nm}$. Silicon is also observed with $35 \%$ atomic concentration on the surface. The silicon concentration decreases slightly as the depth increases to $30 \mathrm{~nm}$ and then increases sharply to $92 \%$ at $40 \mathrm{~nm}$. After $41 \mathrm{~nm}$ the concentration remains almost constant at $98 \%$ up to $160 \mathrm{~nm}$. As it was explained earlier, a sharp decrease of carbon and platinum concentrations that corresponds to a sharp increase of silicon indicates that there is no interface layer formed between platinum and silicon. A presence of silicon on the surface shows that it was exposed when platinum was diffusing into the bulk.

The platinum peak has been reduced from $55 \%$ to $35 \%$ and shifted to $20 \mathrm{~nm}$ after diffusion. This reduction of the peak indicates that the platinum concentration of platinum has been reduced on the surface while the shifting to higher sputter depth shows that platinum has diffused into the bulk of the silicon.

\subsection{Erbium-deposited samples}

Figure 3 shows the XPS spectra for erbium-deposited samples (a) prior to and (a) after the diffusion process. In Fig. 3a the spectra show that carbon with $47 \%$ atomic concentration and oxygen with $40 \%$ atomic concentration dominate on the surface of the silicon. The carbon concentration decreases sharply to $6 \%$ at the sputter depth lower than $2 \mathrm{~nm}$ to show that it is very thin on the surface. After $2.5 \mathrm{~nm}$, the spectrum shows an oscillation between 0 and $5 \%$ carbon concentration until it disappears completely at $20 \mathrm{~nm}$. This oscillation could be due to fluctuation in the XPS system. Unlike in the case of gold-deposited and platinum- deposited samples, the oxygen concentration in this case decreases to $25 \%$ and then remains almost constant up to the sputter depth of $12.5 \mathrm{~nm}$. After $13 \mathrm{~nm}$ the concentration decreases to $5 \%$ at $15.5 \mathrm{~nm}$ and then to $0 \%$. This behaviour suggests that there is a thick layer of oxygen on the surface. Even though the XPS results show the high relative concentration of oxygen on the surface, it has to be noted that the sample was contaminated after the deposition since the oxygen peak was not observed in the RBS measurements [18]. These results show that erbium reacts vigorously with oxygen.

The erbium concentration increases sharply from $11 \%$ on the surface to $65 \%$ at the sputter depth less than $2 \mathrm{~nm}$. After the depth of $2.5 \mathrm{~nm}$ the spectrum shows an oscillation between 74 and $65 \%$ erbium concentration up to the depth of $12 \mathrm{~nm}$. After $12.5 \mathrm{~nm}$ the concentration decreases sharply to $5 \%$ at $20 \mathrm{~nm}$ and then becomes $0 \%$ after $21 \mathrm{~nm}$. 

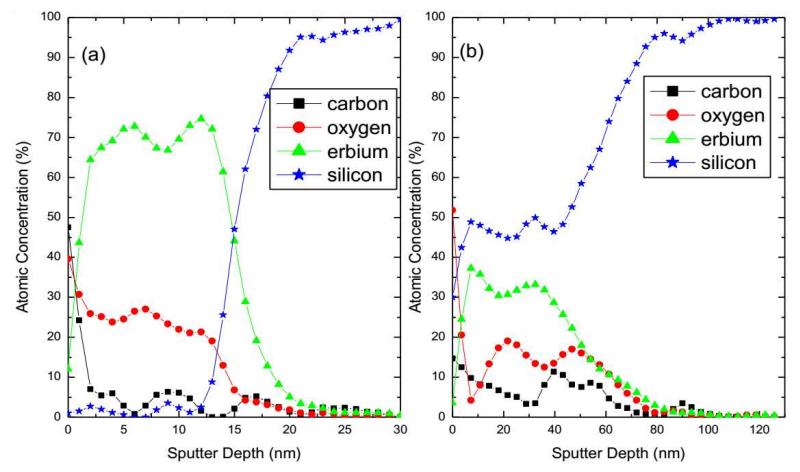

Fig. 3. XPS depth profile for erbium-deposited silicon samples prior to (a) and after (b) the diffusion process.

Figure $3 \mathrm{a}$ shows the $0 \%$ atomic concentration of silicon on the surface. This indicates that silicon has not been exposed to the surface. At the depth of less than $11.5 \mathrm{~nm}$ the spectrum shows an oscillation of silicon concentration between 0 and $3 \%$. This oscillation as it was said before could be due to fluctuation in the XPS system. At the depth higher than $12 \mathrm{~nm}$ the sharp increase of silicon concentration corresponds to the sharp decrease of erbium concentration to show that there is no interface layer formed between erbium and silicon.

In Fig. 3b the spectra show carbon with $15 \%$ atomic concentration and oxygen with $53 \%$ atomic concentration on the surface. The carbon concentration decreases steadily to $3 \%$ at $25 \mathrm{~nm}$. After $30 \mathrm{~nm}$ the concentration increases to $11 \%$ at $40 \mathrm{~nm}$ and then decreases steadily until it becomes $0 \%$ at $70 \mathrm{~nm}$. At the depth less than $7 \mathrm{~nm}$ the oxygen concentration decreases to $4.5 \%$ and then increases to $20 \%$ at $20 \mathrm{~nm}$. After $21 \mathrm{~nm}$ the spectrum shows an oscillation between $15 \%$ and $18 \%$ of oxygen concentration up to $48 \mathrm{~nm}$ and then decreases to $4 \%$ at $78 \mathrm{~nm}$ until it becomes $0 \%$ at $80 \mathrm{~nm}$. Spectra show that carbon and oxygen concentration becomes zero after the longer sputter depth. This indicates that carbon and oxygen are thick on the surface of the sample.

Erbium is found on the surface with the concentration of $3.5 \%$. The erbium concentration increases to $37 \%$ at the depth of less than $7 \mathrm{~nm}$ and then decreases to $28 \%$ at the depth of $18 \mathrm{~nm}$ where it remains almost constant up to $33 \mathrm{~nm}$. After $34 \mathrm{~nm}$ the concentration decreases rapidly to $2.5 \%$ at the depth of $80 \mathrm{~nm}$ and then becomes zero. The silicon spectrum, on the other hand, shows $30 \%$ atomic concentration on the surface indicating that silicon was exposed when erbium was diffusing into the bulk. At the depth less than $7 \mathrm{~nm}$ the concentration increases to $49 \%$ and then oscillates between $50 \%$ and $45 \%$ up to $40 \mathrm{~nm}$. After $41 \mathrm{~nm}$ the concentration increases sharply to $95 \%$ and then saturates. The sharp increase of silicon concentration corresponds to the sharp decrease of erbium concentration to show that there is no interface layer formed between them.

The highest peak of erbium has been reduced from $66 \%$ to $31 \%$ and shifted to a higher sputter depth after diffusion. This reduction of the peak indicates that erbium has been reduced on the surface while the shifting shows that platinum has diffused into the bulk of silicon. Unlike in the case of gold and platinum, the spectrum of erbium is lower than that of silicon indicating that the concentration of erbium diffused is low in all sputter depths.

\subsection{Niobium-deposited samples}

Figure 4 shows the XPS spectra for niobium-deposited samples (a) prior to and (b) after the diffusion process. In Fig. 4a the spectra show carbon with $71 \%$ atomic concentration and oxygen with $25 \%$ atomic concentration dominating on the surface. The carbon concentration decreases sharply to $5 \%$ at the depth lower than $2.5 \mathrm{~nm}$ to show that it is very thin on the surface. After $2.5 \mathrm{~nm}$, the concentration increases to $8 \%$ and then it remains almost constant up to $10 \mathrm{~nm}$ until it becomes $0 \%$ at higher sputter depth. The oxygen concentration increases to $55 \%$ at the depth lower than $2.5 \mathrm{~nm}$. At the depth higher than $2.5 \mathrm{~nm}$ the concentration decreases to $2 \%$ until it becomes $0 \%$ at the depth higher than $8 \mathrm{~nm}$. These results show that oxygen and carbon are thick on the surface.

A very low relative atomic concentration of niobium is observed on the surface. The concentration increases to $51 \%$ at the depth lower than $7 \mathrm{~nm}$ and then decreases to $3 \%$ at the depth of $13 \mathrm{~nm}$. After $13 \mathrm{~nm}$ the concentration remains constant up to $30 \mathrm{~nm}$. It can be noted in Fig. 4a that there is a very low atomic concentration of silicon on the surface. After $2.5 \mathrm{~nm}$ the silicon concentration increases to $5.5 \%$ and then remains constant up to $6 \mathrm{~nm}$. At the depth higher than $6 \mathrm{~nm}$ the concentration increases sharply to $86 \%$ at $13 \mathrm{~nm}$ and then increases gently to $93 \%$ at $17.5 \mathrm{~nm}$. The rapid increase of silicon concentration corresponds to the sharp decrease of niobium concentration to show that there is no interface layer formed between them. After $17.5 \mathrm{~nm}$ the silicon concentration remains almost constant at $95 \%$ up to $30 \mathrm{~nm}$. A decrease in carbon concentration is not rapid after $7.5 \mathrm{~nm}$ indicating that is found in the bulk.
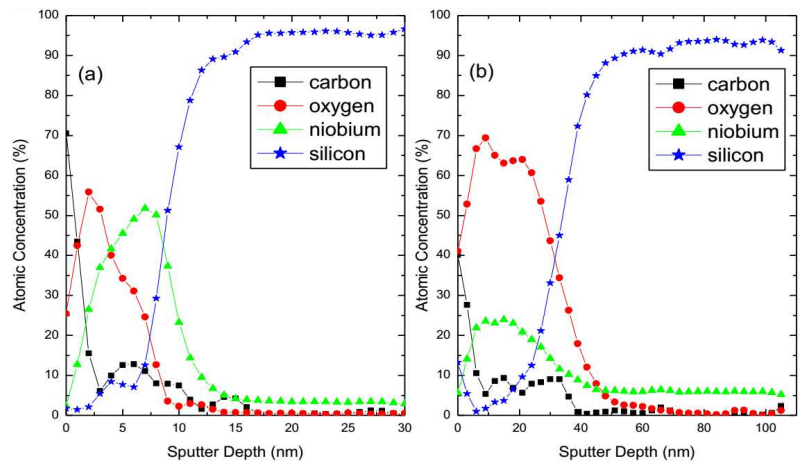

Fig. 4. XPS depth profile for niobium-deposited samples prior to (a) and after (b) the diffusion process.

In Fig. 4b the spectra show carbon with $40 \%$ atomic concentration and oxygen with $41 \%$ atomic concentration on the surface. The carbon concentration decreases 
rapidly to $5 \%$ at $9 \mathrm{~nm}$ and oscillates between 5 and $10 \%$ up to $32 \mathrm{~nm}$. After $32 \mathrm{~nm}$ the concentration becomes $0 \%$. At a depth of less than $10 \mathrm{~nm}$ the oxygen concentration increases sharply to $70 \%$ and then decreases to $64 \%$ at $22.5 \mathrm{~nm}$. After $23 \mathrm{~nm}$ the concentration decreases sharply to $3 \%$ at $50 \mathrm{~nm}$ and then becomes $0 \%$. The concentration of oxygen has increased from 53 to $70 \%$ and shifted from $7 \mathrm{~nm}$ to $18 \mathrm{~nm}$ after diffusion process. This may suggest that niobium reacts vigorously with oxygen.

Niobium is found on the surface with the relative concentration of $5 \%$. The niobium concentration increases to $24 \%$ at the depth less than $10 \mathrm{~nm}$ and then remains almost constant up to $18 \mathrm{~nm}$. After $19 \mathrm{~nm}$ the concentration decreases gently to $8 \%$ at $40 \mathrm{~nm}$ and then it remains constant. The silicon spectrum, on the other hand, shows $14 \%$ atomic concentration on the surface to show that it was exposed when niobium was diffusing into the bulk. At the depth less than $6 \mathrm{~nm}$ the silicon concentration decreases sharply to $0 \%$ and then increases gently up to $15 \%$ at $18 \mathrm{~nm}$. After $20 \mathrm{~nm}$ the concentration increases sharply to $92 \%$ at $50 \mathrm{~nm}$ and then remains constant. The sharp increase of silicon spectrum corresponds to the sharp decrease of oxygen and niobium spectra to show that there is no interface layer formed between niobium and silicon.

It can be noted that niobium peak has been reduced from $51 \%$ to $25 \%$ and shifted to a higher sputter depth of $10 \mathrm{~nm}$ after diffusion process. This reduction of the peak indicates that niobium has reduced on the surface while the shift to higher sputters depth shows that it has diffused into the bulk.

\section{Overall discussions}

XPS measurements were carried out on metaldeposited samples prior to and after the diffusion process. This work adds on that presented on RBS results presented earlier $[10,18]$ since the diffusion of metals was speculated. The results presented here are thus used to explain features that could not be explained by the RBS data and also confirm that metals diffuse in silicon at $900{ }^{\circ} \mathrm{C}$ for $18 \mathrm{~h}$ of annealing. The highest peaks of gold, platinum and niobium are found to be higher than that of silicon indicating that the metals are found with considerable atomic concentration at the sputter depth of $20 \mathrm{~nm}$ in silicon. The results indicate that gold, platinum and niobium diffuse with more considerable amount into silicon at an annealing temperature of $900{ }^{\circ} \mathrm{C}$ if the process is done over $18 \mathrm{~h}$. At this annealing temperature and over this time the diffusion profile of gold and platinum in silicon is U-shaped and is dominated by the kick-out [2022 . It has to be noted that the diffusion mechanisms of niobium are currently not known.

The erbium spectrum, on the other hand, is lower than that of silicon in the whole sputter depth. This indicates that the amount of diffused erbium is low. This is due to a very low diffusion coefficient that the metal has in silicon at $900^{\circ} \mathrm{C}$ [23]. The diffusion profile of erbium in silicon is currently not well understood. It has been reported that at the annealing temperature of $900^{\circ} \mathrm{C}$ for $30 \mathrm{~min}$, erbium likely diffuses interstitially in silicon [24]. Contrary, it was also reported that most of erbium atoms in silicon occupy substitutional sites [25]. These contradictory studies indicate that there is no definite answer about the diffusion mechanisms of erbium in silicon.

\section{Conclusion}

The results from the XPS measurements show that there is no interfacial layer formed between silicon and the deposited metal layer. The results also show that the spectrum peak of metal-doped samples gets reduced and shifted towards higher depths after the diffusion process. This reduction of the peak heights indicates that the amount of metals on the silicon surface has been reduced. The shift of the peak indicates a presence of metal in the silicon bulk. Both results show that the metal from the surface of the silicon has diffused into the bulk. These diffused metals have been shown to render the silicon material relaxation-like. The XPS technique can thus be used to complement the RBS technique in depth profiling of our defect-engineered silicon.

\section{Acknowledgments}

We thank M. Msimanga at Tshwane University of Technology for assistance with preparation of the samples and the CSIR for availing their PEQ 2000 instrument to us.

\section{References}

[1] M. Martini, T.A. McMath, Nucl. Instrum. Methods 79, 259 (1970).

[2] V.A.J. Van Lint, Nucl. Instrum. Method Phys. Res. A 253, 453 (1987).

[3] M. Moll, H. Feick, F. Fretwurst, G. Lindstrom, C. Schutze, Nucl. Instrum. Methods Phys. Res. A 388, 335 (1997).

[4] U. Gosele, W. Frank, A. Seeger, Appl. Phys. 23, 361 (1980).

[5] R.L. Dixon, K.E. Ekstrand, Radiat. Protect. Dosim. 17, 527 (1986).

[6] Y.K. Kwon, T. Ishikawa, H. Kuwano, J. Appl. Phys. 61, 1055 (1987).

[7] J.L. Benton, J. Michel, L.C. Kimerling, D.C. Jacobson, Y.H. Xie, D.J. Eaglesham, E.A. Fitzgerald, J.M. Poate, J. Appl. Phys. 70, 2667 (1991).

[8] H. Pettersson, Grimmeiss, L. Tilly, Schalz, H. Kerkow, Semicond. Sci. Technol. 8,1247 (1993).

[9] M. McPherson, T. Sloan, B.K. Jones, J. Phys. D Appl. Phys. 30, 3028 (1997).

[10] M. Msimanga, M. McPherson, Mater. Sci. Eng. B 127, 47 (2006).

[11] S.J. Moloi, M. McPherson, Physica B 404, 2251 (2009).

[12] W. van Roosbroeck, Phys. Rev. 123, 474 (1961).

[13] H.J. Queisser, IOP Conf. Series 15, 145 (1972).

[14] M. Ilegems, H.J. Queisser, Phys. Rev. B 12, 1443 (1975). 
[15] N.M. Haegel, Appl. Phys. A 53, 1 (1991).

[16] V.N. Brudnyi, S.N. Grinyaev, V.E. Stepanov, Physica B 212, 429 (1995).

[17] M. McPherson, Physica B 344, 52 (2002).

[18] S.J. Moloi, M. McPherson, Vacuum 104, 51 (2014).

[19] J.W. Pankow, Surface Analysis of Stressed and Control Tin Oxide Thin Films on Soda Lime Glass, presented at the National Centre for Photovoltaics and Solar Program Review Meeting, Denver (USA), 2003.

[20] F. Morehead, N.A. Stolwijk, W. Meyberg, U. Gosele, Appl. Phys. Lett. 42, 690 (1983).

[21] S. Coffa, L. Calcagno, S.U. Campisano, G. Calleri, G. Ferla, J. Appl. Phys. 64, 6291 (1988).
[22] O. Boström, B. Pichaud, M. Regula, J.C. Bajard, G. Blondiaux, O.A. Soltanovich, E.B. Yakimov, A. Lhorte, J.B. Quoirin, Mater. Sci. Eng. B 71, 166 (2000).

[23] J.H. Shin, G.N. van den Hoven, A. Polman, Appl. Phys. Lett. 67, 377 (1995).

[24] M. Needels, M. Schlüter, M. Lannoo, Phys. Rev. B 47, 15533 (1993).

[25] Y.S. Tang, Zhang Jingping, K.C. Heasman, B.J. Sealy, Solid State Commun. 72, 991 (1989). 